\title{
On the role of hydrogen bond strength and charge transfer of a Diels-Alder reaction On-Water: Semiempirical and free energy calculations.
}

\author{
Andrés Henao, ${ }^{1, a)}$ Yomna Gohar, ${ }^{1,2}$ René Wilhelm, ${ }^{3}$ and Thomas D. Kühne ${ }^{1,4, b)}$ \\ ${ }^{1)}$ Dynamics of Condensed Matter, Chair of Theoretical Chemistry, University of Paderborn, Warburger Str. 100, \\ D-33098 Paderborn, Germany \\ ${ }^{2)}$ current address: International Max Planck Research school, George August University in Goettingen, Wilhelmsplatz 1, \\ 37073 Goettingen, Germany \\ 3) Intitute of Organic Chemistry, Clausthal University of Technology, Leibnizstr. 6, D-38678 Clausthal-Zellerfeld \\ 4) Paderborn Center for Parallel Computing and Institute for Lightweight Design, University of Paderborn, Warburger Str. 100, \\ D-33098 Paderborn, Germany
}

(Dated: 11 April 2021)

Accelerated chemistry at the interface with water has received increasing attention. The mechanisms behind the enhanced reactivity On-Water are not yet clear. In this work we use a Langevin scheme in the spirit of second generation Car-Parrinello to accelerate the second-order density functional Tight-Binding (DFTB2) method in order to investigate the free energy of two Diels-Alder reaction On-Water: the cycloaddition between cyclopentadiene and ethyl cinnamate or thionocinnamate. The only difference between the reactants is the substitution of a carbonyl oxygen for a thiocarbonyl sulfur, making possible the distinction between them as strong and weak hydrogen-bond acceptors. We find a different mechanism for the reaction during the transition states and uncover the role of hydrogen bonds along with the reaction path. Our results suggest that acceleration of Diels-Alder reactions do not arise from an increased number of hydrogen bonds at the transition state and charge transfer plays a significant role. However, the presence of water and hydrogen-bonds is determinant for the catalysis of these reactions.

\section{INTRODUCTION:}

Water is of paramount importance to life; its role in the biological processes has been long recognized. However, its use in chemistry is less established as many substances of interest are not soluble in water, which has relegated water use in chemical reactions. The discovery of the acceleration of Diels-Alder reactions on the interface with water in $1980^{1}$, coined later as on-water ${ }^{2}$ catalysis, has changed this panorama. Recently more and more examples of the acceleration of chemical processes at the interface with water have been described ${ }^{3-7}$.

The mechanism under which reactions at aqueous interfaces accelerate remains unclear ${ }^{8}$. Firstly, a theory of solvation at interfaces is more complex than bulk solvation and a current research topic. Secondly, the wide variety of phenomena occurring at aqueous interfaces may imply different mechanisms, making the interpretation of experimental measurements a complex task. Computational models are highly desirable to understand experiments and elucidate the underlying mechanisms driving the reactions at aqueous interfaces. Jung and Marcus ${ }^{9}$ proposed a mechanism for the on-water catalysis based on the availability of free $\mathrm{OH}$ bonds at the interface, interacting through hydrogen bonds with the molecular species. This oppositely to the homogeneous inwater case, where hydrogen bonds require higher energy to be broken and are therefore less available. Yet, in-water reactions can be accelerated due to the Breslow hydrophobic

\footnotetext{
a)Electronic mail: andresh@mail.uni-paderborn.de, ahenaoa@unal.edu.co

b)Electronic mail: tdkuehne@mail.uni-paderborn.de
}

effect $^{10,11}$. Other mechanisms have also been proposed: Recently, an experimental study ${ }^{12}$ found an additional increase in the reaction rate of diethyl azodicarboxylate (DEAD) and quadricyclane $(\mathrm{Q})$ cycloaddition when performed on microdroplets compared to the on-water reaction, which they coined as "on-droplet" chemistry. This study pointed at the effect of the surface-to-volume ratio and supported the acid-catalysis mechanism of Beattie and co-workers ${ }^{13}$, where reaction with water at the interface results in both the protonated substrate and free $\mathrm{OH}^{-}$, which is stabilized by its strong adsorption at the interface. Finally, a recent review comprises the contradictory and unexplained mechanisms. It proposes a revised on-water model ${ }^{14}$ that conceives that the interfacial water molecules would undergo partial polarization because of a loss of balance between donation and acceptance of hydrogen bonding through the Grotthuss mechanism at the interface, as a modified "on water" model.

The different mechanisms proposed and the wide variety of reaction mechanisms accelerated at the interface with water highlight the importance of using computational methods to shed light on the catalysis mechanism at water interfaces. The systems studied have been, however, limited by the computational feasibility. The use of improved methodologies, which allow us to treat accurately but efficiently the required degrees of freedom to describe the reactions at realistic heterogeneous interfaces, is therefore of great importance to uncover the interplay of hydrogen bonds and reaction accelerations on-water. Previous attempts to study on-water reactions have shown some insights. A lowering of the free energy barrier has been reported for a solvated retro-Diels-Alder reaction using 50 water molecules (in-water) in comparison to the vapor phase reaction. The authors used Car-Parrinello molecular dynamics ${ }^{15}$ and concluded that water solvent is pivotal to this 


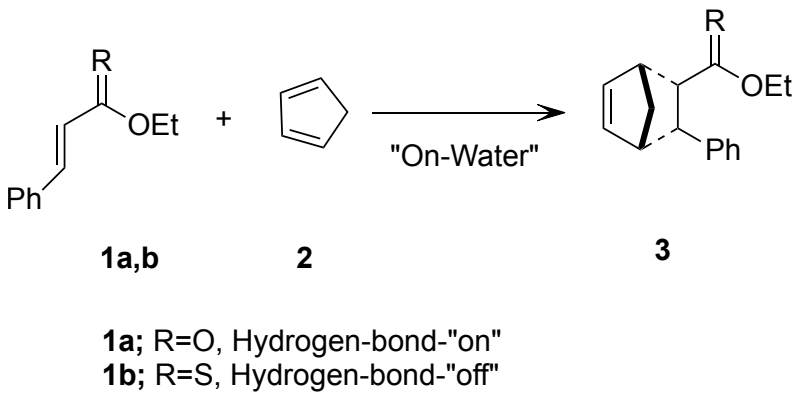

FIG. 1. Diels-Alder reaction with cinnamates (1) and cyclopentadiene (2) on the interface with water. When the carbonyl oxygen in ethyl cinnamate (1a or Hydrogen-bond-"on") is replaced by a thiocarbonyl sulfur, a thiocnocinnamate (1b or Hydrogen-bond-"off") is obtained. The product $\mathbf{3}$ shows the new formed bonds (dashed bonds) between $\mathbf{1}$ and $\mathbf{2}$ The average of these two new formed bonds will be used as reaction coordinate for the free energy calculations.

effect, especially for compounds containing an electron donating substituent. The role of microsolvation has been similarly highlighted, Pestana et al. ${ }^{11}$ used ab-initio simulations to investigate a retro Diels-Alder reaction in bulk water under different confinements and found the acceleration to be similar. Therefore, the deciding factor is the microsolvation, suggesting that no additional catalysis would be expected for on-water via a local hydrogen-bond mechanism. Karhan et al. ${ }^{16}$ compared the homogeneous and heterogeneous DielsAlder reaction between dimethyl azodicarboxylate (DMAD) and quadricyclane $(\mathrm{Q})$. They found that the number of hydrogen bonds for the transition state and reactants is similar and is not increased as expected from Jung and Marcus ${ }^{9}$. Recently Salem and Kühne investigated the DMAD+Q reaction using an energy decomposition analysis of the reactants, transition state and product in the presence of three water molecules. They found that charge transfer plays an important role ${ }^{17}$. Yang and co-workers also recently highlighted the role of charge transfer using an ab-initio quality neural network to model a cycloaddition reaction on-water ${ }^{18}$.

We attempt to investigate the importance of hydrogen bond strength for accelerating an on-water Diels-Alder reaction, inspired by an experimental study on the Diels-Alder reaction with ethyl crotonthioate as dienophile ${ }^{19}$. We study two systems shown in Figure 1, where one of the reactants is slightly different and forms bonds with cyclopentadiene: The first one is ethyl cinnamate containing a carbonyl oxygen, which we will call H-bond-"on" from now on. The second one is a thionocinnamate ${ }^{19}$, where thiocarbonyl sulfur replaces the carbonyl oxygen. As sulfur has a weaker hydrogen-bond interaction with water ${ }^{19}$, we will call this the H-bond-"off" system.

\section{COMPUTATIONAL DETAILS}

The modeling of a realistic heterogeneous interface is an important ingredient to investigate the effect of the water interface on the acceleration of reactions. We have therefore used the second-order density functional tight-binding ${ }^{20}$ (DFTB2) approach as implemented in $\mathrm{CP} 2 \mathrm{~K}^{21}$ version 7.1. This method allows us to approximately treat the quantum nature of the electrons by solving self-consistently the charge density. The efficiency of DFTB2 lies in between Density Functional Theory and classical molecular dynamics. Even-though systems on the order of thousand atoms are now feasible with DFTB2, the evaluation of the free energy of the chemical reactions at the interface requires several simulations sampling different points along a reaction coordinate. To make this feasible, we have used a linear scaling technique inspired by the Second-Generation-Car-Parrinello scheme ${ }^{22}$, where the numerical precision is reduced, and a Lagrange scheme $\mathrm{e}^{23}$ is used to compensate for this, improving the efficiency while keeping the accuracy of the self-consistent charge densities. This allowed us to perform more than 70 simulations efficiently for the two systems of interest and obtain the free energy profile of the on-water reactions. Each system consisted of 22 cyclopentadiene molecules, 4 cinnamates, and 278 water molecules. The reactant molecules were chosen close to the interface with water, and they remained there during the simulation time. We used the original mio-1-1 parameters ${ }^{20}$ for the organic molecules and the modified parametrization ${ }^{24}$ for water.

Free energy calculations were done using an umbrella sampling potential $\mathrm{k}\left(\xi-\xi_{0}\right)^{2}$ along with the reaction coordinate $\xi$. This reaction coordinate was chosen as the average distance of the two bonds that are formed in the Diels-Alder reaction, shown in Figure 1. The free energy was evaluated along this coordinate between $4.5 \AA$, namely the reactants, and $1.5 \AA$, namely the product, every $0.1 \AA$ with a force constant of $\mathrm{k}=470 \mathrm{Kcal} / \mathrm{mol} / \AA^{2}$. Four additional umbrella windows were simulated close to the identified transition states with a tighter force constant of $\mathrm{k}=1880 \mathrm{Kcal} / \mathrm{mol} / \AA^{2}$ and every $0.05 \AA$, two at each side of the transition state. The weighted histogram analysis method ${ }^{25}$ was employed to recover the unbiased probabilities using the WHAM $^{26}$ implementation.

\section{RESULTS}

The use of the linear-scaling technique along the DFTB2 method was first tested to assess its accuracy at obtaining free energy profiles. For this, a set of short $10 \mathrm{ps}$ was performed to obtain the full energy profile using the DFTB2 method alone. The self-consistent charge distribution was obtained using the Orbital-Transformation ${ }^{27}$ and an SCF accuracy of $10^{-7}$. The resulting free energy was then compared to a set of equally long (10 ps) simulations using the Langevin approach at an $\mathrm{SCF}=10^{-6}$ resulting in a 4-times increased efficiency. Figure 2 compares the relative free energies, which are the same within the statistical uncertainty (error sampling was obtained using the bootstrap analysis implemented in $\mathrm{WHAM}^{26}$ ), proving that the Langevin-DFTB2 method provides accurate estimates of free energies at a reduced computational cost. Therefore, Langevin-DFTB2 was employed to obtain 100 ps simulations at 35 different windows along with the reaction coordinate for both H-bond-"on" and -"off" systems. These win- 


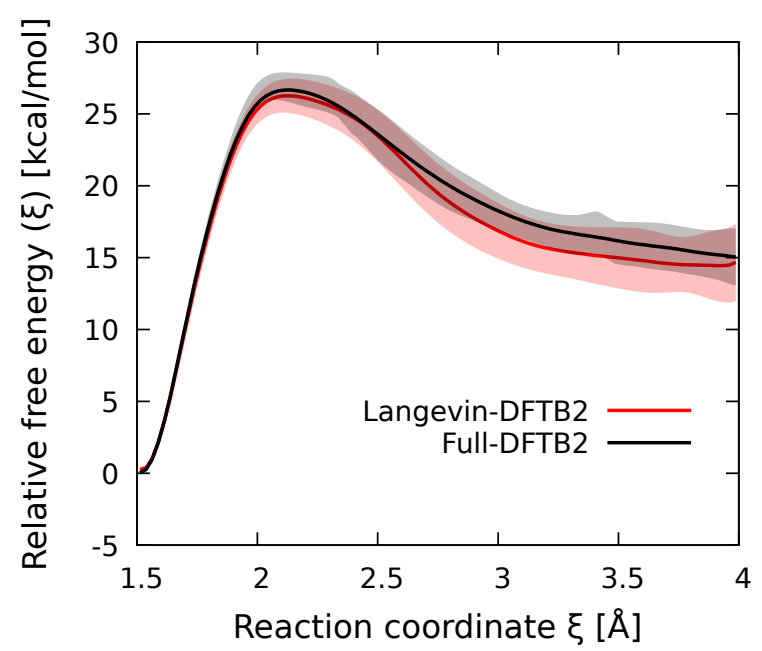

FIG. 2. Color online. Relative free energy profiles obtained from the Full-DFTB2 using OT (black curve) and the accelerated LangevinDFTB2 scheme (red curve). Umbrella sampling simulations during $10 \mathrm{ps}$ were used, and the free energies obtained using $\mathrm{WHAM}^{26}$. The errors were estimated using the bootstrap analysis, shown as a shaded filled area for each curve. These results show that Langevin-DFTB2 produces the same results for the free energies within the statistical error at a reduced computational cost.

dows were sampled every $0.1 \AA$, and four additional windows close to the transition states were used with a $0.05 \AA$ precision.

The relative free energy profiles are shown in Figure 3 for both the H-bond-"on" (red curve) and -"off" (yellow curve) systems. The first difference that can be traced is that of the shape of the free energy profiles; this indicates a different activation of the cycloaddition reaction even-though both systems differ only on the carbonyl $\mathrm{C}=\mathrm{O}$ and thiocarbonyl $\mathrm{C}=\mathrm{S}$ groups, which do not participate directly in the formation of the new bond. The energy barrier of formation is obtained as the difference in the relative free energies between the reactants (at reaction coordinates around $4.5 \AA$ ) and the transition state (TS). In the case of the H-bond-"on" system, the TS is located at $2.1 \AA$ and the energy barrier is $13 \mathrm{Kcal} / \mathrm{mol}$, while the TS for the H-bond-"off" system is located around $1.9 \AA$ and the energy barrier is $24 \mathrm{Kcal} / \mathrm{mol}$. This shows a larger penalty for the Diels-Alder reaction of the H-bond-"off" occurring at the water interface. Another clear difference arises for the two reactions; this is the free energy of formation of the products $\Delta \mathrm{A}=A_{\text {product }}-A_{\text {reactant }}$. This $\Delta A<0(-14 \mathrm{Kcal} / \mathrm{mol})$ in the H-bond-"on" system and $\Delta A>0(+7 \mathrm{Kcal} / \mathrm{mol})$ in the H-bond-"off" system, indicating an exothermic and endothermic reaction for each system respectively. Next, the umbrella sampling trajectories will be analyzed to obtain atomistic insights into the mentioned differences.

An inspection of the trajectories for each umbrella sampling simulation at the window corresponding to the transition states, at $2.1 \AA$ and $1.9 \AA$ is shown in Figure 4 . A representative snapshot of the TS of both systems revealed a difference: The bond formation occurs in an asynchronous way in both systems; this is the two $\mathrm{C}-\mathrm{C}$ bonds are not simultaneously created. For both systems the first $\mathrm{C}-\mathrm{C}$ bond is

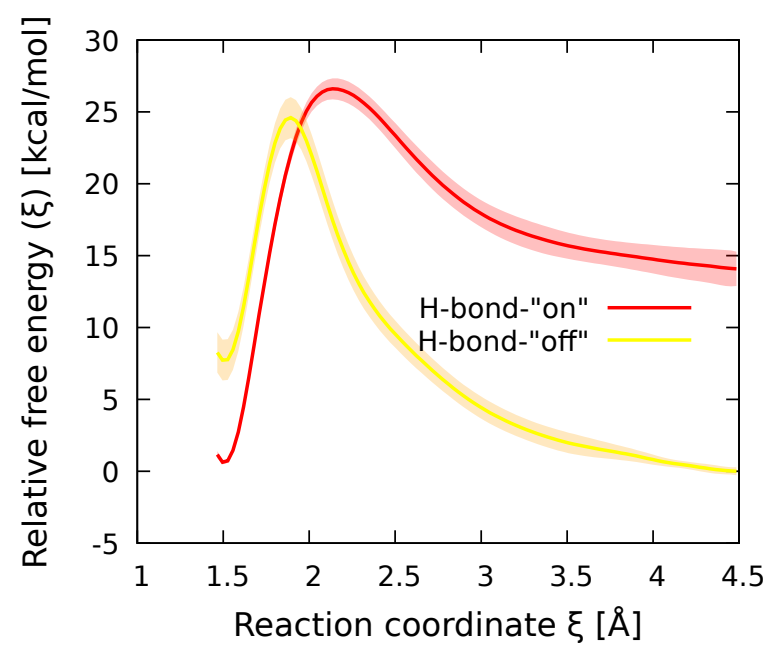

FIG. 3. Color online. Relative free energy profiles obtained from Umbrella sampling simulations using the accelerated LangevinDFTB2 scheme during 100 ps for the H-bond-“on" (red curve) and H-bond-"off" (yellow curve). The shaded area shows the statistical errors as estimated using the bootstrap analysis implemented in WHAM $^{26}$.

created and stabilized between cyclopentadiene and the cinnamate (see Fig. 1). However, the second C-C bond is formed in the H-bond-"on" system relatively easy as it occurs in a single step. This is not the case for the H-bond-"off" system, where a transient bond is observed in Fig. $4 \mathrm{~b}$ between sulfur and a carbon atom in cyclopentadiene 28,29 . This implies that the formation of the second $\mathrm{C}-\mathrm{C}$ bond has to overcome an additional energy barrier in the H-bond-"off" system due to an interaction between sulfur and cyclopentadiene. This reveals that the carbonyl $\mathrm{C}=\mathrm{O}$ species plays a stabilizing role during the approach of the reactants at the interface with water, while the thiocarbonyl $\mathrm{C}=\mathrm{S}$ species interferes with the formation of the second C-C bond. This could be attributed probably to the stronger electronegativity of oxygen compared to sulfur and its ability to form stronger hydrogen bonds with water. However, further analyses are necessary to understand this mechanism. We have quantified the asynchronicity of transition state as a way to characterize the mechanism of the Diels-Alder reaction, defined as the difference in length of the newly formed bonds between $\Delta \mathrm{C}=\mathrm{C}_{\mathrm{bond}}^{1}-\mathrm{C}_{\mathrm{bond}}^{2}$. This value is small for the H-bond-"on" system and large for the H-bond-"off" system, $\Delta \mathrm{C}$ "on" $=0.02 \pm 0.20 \AA$ and $\Delta \mathrm{C}$ "off" $=0.95 \pm 0.07 \AA$. This indicates that the reaction happens synchronously in the strong H-bonded system, and it is asynchronous in the weak H-bonded system, highlighting a different reaction mechanism for these systems as observed in the different shape of free energy profiles in Fig. 3.

The bond formation can be qualitatively traced using a charge population analysis at different steps during the reaction. As a self-consistent redistribution of Mulliken charges modifies the Hamiltonian matrix elements in DFTB2, we used the Mulliken population analysis ${ }^{30}$ in this work. While there are other schemes, such as DDEC ${ }^{31,32}$, Mulliken charges provide a qualitative picture sufficient within the approximations 


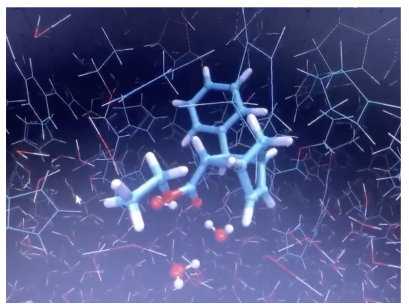

(a) H-bond-"on" transition-state

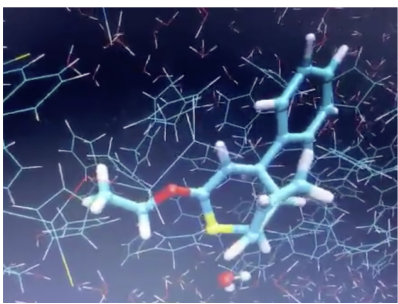

(b) H-bond-"off" transition-state
FIG. 4. Color online. Snapshot of the transition states (TS). The formation of the second $\mathrm{C}-\mathrm{C}$ bond is more stable in the H-bond-"on" system. The TS of the H-bond-"off" revealed the transient formation of a further S-C bond between the thionocinnamate and cyclopentadiene. This competition effect against the formation of the second $\mathrm{C}-\mathrm{C}$ bond is responsible for the different relative free energy profile and higher energy barrier shown in Fig. 3

already made by the choice of the DFTB2 method. Results are shown in Figure 5. We recovered the unbiased charges $P(q)$ applying the corresponding reweight to the biased charges $P^{\prime}(q)$ according to the form:

$$
P(q) \propto P^{\prime}(q) \exp \left(\frac{\mathrm{k}\left(\xi-\xi_{0}\right)^{2}}{k_{\mathrm{B}} T}\right)
$$

We have inspected the charge population for six different groups: The two carbon atoms directly involved in the cycloaddition reaction, shown in Figs. 5a and 5b, which show a different charge population evolution on the second carbon atom for the H-bond-"off" system as suggested by the transition states trajectories and asynchronicity. Next, Fig. 5c shows the carbonyl oxygen and thiocarbonyl sulfur atoms. In this group of atoms, a big difference is observed. On the one hand, the oxygen atom has a constant charge along the reaction path, while on the other hand, sulfur has a decrease of charge around the transition state. This is correlated to the transient bond formation observed in the transition state trajectories between sulfur and cyclopentadiene. Also, oxygen is more electronegative $(\sim-0.6 e)$ than sulfur $(\sim-0.4 e)$ which makes it a better electron hydrogen-bond donor. The charge of the carbon atom bonded to the previous $\mathrm{O}$ and $\mathrm{S}$ atoms is presented in Fig. 5d showing no change along the reaction path for both systems. The same is true for Fig. 5e showing the oxygen atom in the ethyl cinnamate and ethyl thionocinnamate $\mathrm{C}-\mathrm{O}-\mathrm{C}$ group, which has a constant charge for both systems. Finally, the total charge of cyclopentadiene shown in Fig. 5f increases from reactant to product and has a maximum which is correlated with the position of the transition state in the free energy profile (see Fig. 3). The change in the total charge of cyclopentadiene is larger for the H-bond-"on" system. We investigate next the role of hydrogen bonds along with the reaction profile.

An H-bond increase at the transition state has been suggested $^{9}$ as a mechanism to explain the increase of reactivity On-Water. We have investigated the number of hydrogen bonds between water molecules and carbonyl oxygen $\mathrm{C}=\mathrm{O}$ and thiocarbonyl sulfur $\mathrm{C}=\mathrm{S}$ atoms, in fact the hydrogen bonds of water with reactants occur through these atoms only. We applied the same weight shown in eq. 1 to obtain the unbiased number of hydrogen bonds. These atoms also showed the maximum difference in the charge evolution along the reaction path of Fig. 5c and also the sulfur atom was found to behave differently at the transition state for the H-bond-"off" system (See Fig. 4). Our results (Figure 6) show a different trend for the number of hydrogen bonds for both systems. In the case of the H-bond-"on" reaction, the number of hydrogen bonds remains almost constant (within statistical uncertainty) around a value of 1.2, while the number of hydrogen bonds does change along the reaction path for the H-bond-"off" system: it decreases to almost zero around the transition state (1.9 $\AA$ ), followed by an increase at $1.8 \AA$, finally reaching a value of 0.5 for the product (similar to that of reactants). The evolution of the number of hydrogen bonds in both cases is correlated to the corresponding evolution of the charge of oxygen and sulfur atoms (Fig. 5c): almost constant for oxygen, and with a maximum around the transition state for sulfur, corresponding to a minimum number of hydrogen bonds. This agrees with the observed trajectories of the transition states, where sulfur is also bonded to cyclopentadiene and competes with the formation of the second $\mathrm{C}-\mathrm{C}$ bond. We have also inspected the distribution of hydrogen bond distances and angles in Figures $\mathrm{S} 1$ and $\mathrm{S} 2$ for both systems. We found no evidence for shorter hydrogen bonds in the transition state.

\section{DISCUSSION}

Regarding the role of hydrogen bonds, we have not observed an increase in the number of hydrogen bonds (Fig. 6) for the transition state of H-bond-"on", contrary to the picture of the $\mathrm{H}$-bond mechanism ${ }^{9,33}$. In the case of the $\mathrm{H}$ bond-"off" mechanism, this number decreased at the transition state. We suggest this to be correlated with the increase in the activation energy of the reaction, which is also correlated to the competition of $\mathrm{C}-\mathrm{C}$ bonding with the interaction between sulfur atom and cyclopentadiene. This added a cost to the cycloaddition reaction of the H-bond-"off" system. We propose that the role of hydrogen bonds, and therefore of the water interface, is to stabilize the configurations of the reactants for the formation of new bonds to occur. This has been well contrasted in our results between both systems where the system with a weaker H-bond interaction (sulfur has a smaller electronegativity compared to oxygen) has to overcome a larger energy penalty caused by the changing conformation of the reactants by interacting via the thiocarbonyl sulfur atom. We have not found either, any indication of strengthened hydrogen bonds at the transition states compared to reactants or products. The picture is that, the presence of hydrogen bonds is important as highlighted in the higher penalty for the H-bond-"off" reation $(11 \mathrm{Kcal} / \mathrm{mol}$ compared to $\mathrm{H}$ bond-"on" reation). In this system, we observed a depletion in the number of hydrogen bonds around the transition state in Fig. 6. Cyclopentadiene (CPD) and methyl vinyl ketone (MVK) QM/MM studies of the reaction in water suggested that the $\mathrm{H}$-bond is enhanced at the transition states observ- 


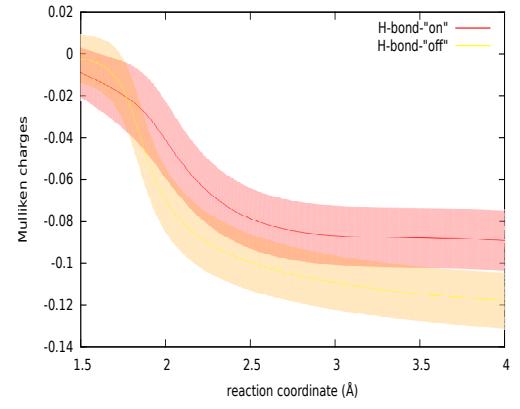

(a) First-Carbon CPD

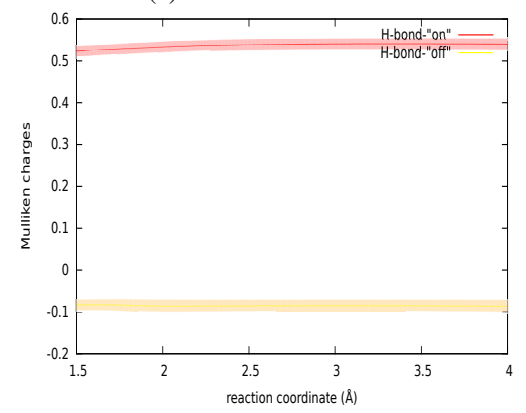

(d) Carbon bonded to $\mathrm{O}$ and $\mathrm{S}$

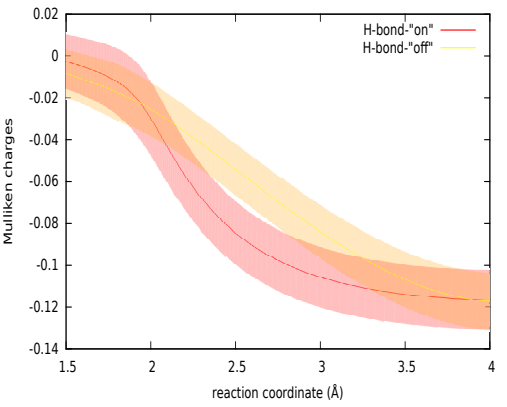

(b) Second-Carbon CPD

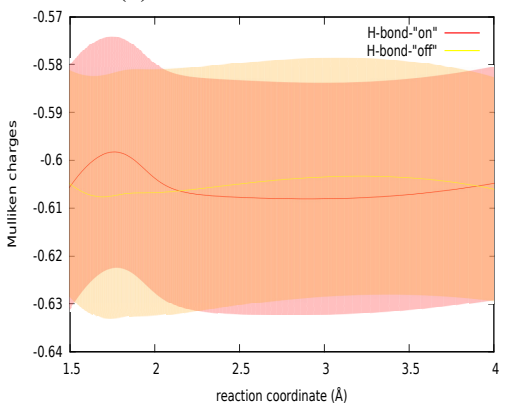

(e) Oxygen C-O-C

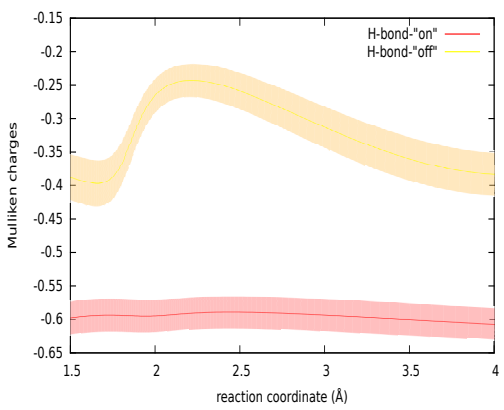

(c) Oxygen and Sulfur

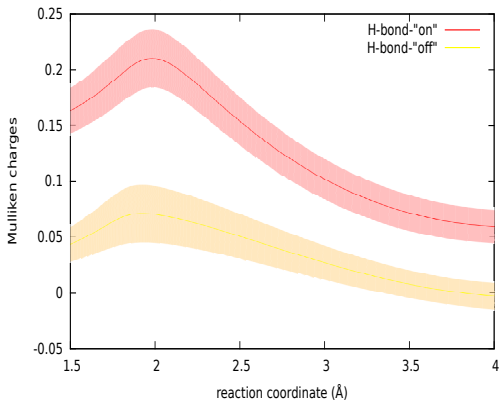

(f) Cyclopentadiene

FIG. 5. Color online. Mulliken charges for different atoms and compared between the H-bond-"on" (red curves) and H-bond-“off" (yellow curves) systems.

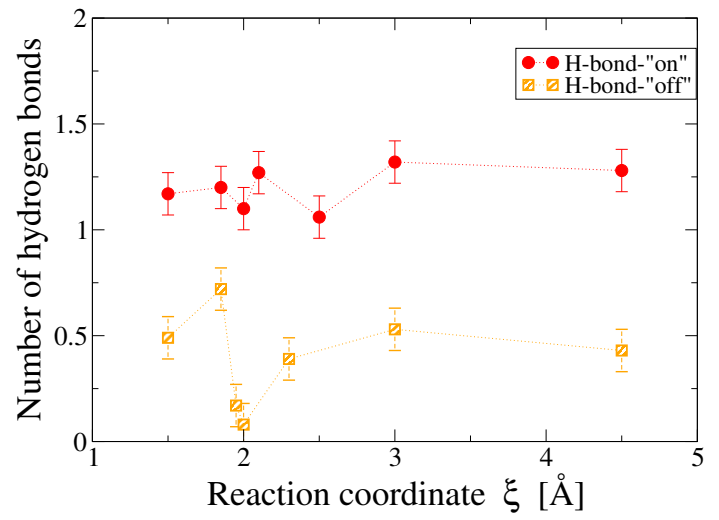

FIG. 6. Color online. The number of hydrogen bonds between water molecules and carbonyl oxygen $\mathrm{C}=\mathrm{O}$, and thiocarbonyl sulfur $\mathrm{C}=\mathrm{S}$ atoms along with the reaction coordinate. Panel (a) shows the results for the H-bond-"on" system. The number of hydrogen bonds remains constant within the error bars along the reaction path. Panel (b) shows the results for the H-bond-"off" system. There is a slight decrease in the number of hydrogen bonds around the transition state.

ing a shortening of the $\mathrm{H}$-bond distance and the role of water reorientation ${ }^{34,35}$, we found however, no evidence of a shortening of hydrogen bonds at the transition state (see Figs. S1 and S2) for the Diels-Alder reaction of this work at the interface with water, or on-water. A similar study using QM/MM MD found enhancement of the same reaction in water compared to methanol ${ }^{36}$. Authors assigned the enhancement from the H-bond formed during the TS between the carbonyl oxygen atom and hydrogen atoms in solvent being responsible to polarize the $\mathrm{C}=\mathrm{O}$ bond and stabilizing the system charge reorganization in the reaction process. We found a similar mechanism from our results on the charge population analysis (see Fig. 5), even though our results are at the interface and not in bulk, this suggests that it is the stabilization of the transition states through a charge redistribution contributing to acceleration of the reactions. The role of charge transfer has also been discussed by other authors ${ }^{17,36}$. A recent study of the water/oil interface found significant build up of surface charge density at hydrophobic interfaces ${ }^{37}$. This is however, not a general statement as we have studied a single reaction and the wide variety of phenomena occurring at aqueous interfaces may imply different mechanisms for other reactions.

\section{CONCLUSIONS}

* No evidence on the increase of the number of hydrogen bonds, neither strengthened hydrogen bonds at the transitionstates as suggested by Jung and Marcus.

* Different mechanism of reaction for the strong hydrogen bonded (synchronous) and weak hydrogen bonded system (asynchronous)

* Role of hydrogen bonds at the water interface is the stabilization of conformations of the reactants. Charge transfer stabilization of the transition states was also observed.

*Correlation between the free energy activation increase of the reaction (H-bonded strong vs weak systems) and the 
number of hydrogen bonds decreasing at the transition state for the weak H-bond system, observed also as a variation on the charge of sulfur atom at the transition state vs a constant charge of the oxygen atom during the complete reaction profile.

*The strength of H-bonds role is suggested to be more important than the number of hydrogen bonds from our study (Strong-Oxygen vs Weak-Sulfur H-bond acceptors).

\section{ACKNOWLEDGMENTS}

The authors gratefully acknowledge the Gauss Centre for Supercomputing e.V. (www.gauss-centre.eu) for funding this project by providing computing time through the John von Neumann Institute for Computing (NIC) on the GCS Supercomputer JUWELS at Jülich Supercomputing Centre (JSC). The generous allocation of computing time on the FPGAbased supercomputer "Noctua" by the Paderborn Center for Parallel Computing $\left(\mathrm{PC}^{2}\right)$ is kindly acknowledged. This project has received funding from the European Research Council (ERC) under the European Union's Horizon 2020 research and innovation programme (Grant Agreement No. 716142). T.D.K. kindly acknowledges funding from Paderborn University's research award for "GreenIT". Finally, A. H. thanks the Alexander von Humboldt Foundation for his postdoctoral research fellowship.

${ }^{1}$ D. C. Rideout and R. Breslow, "Hydrophobic acceleration of diels-alder reactions," Journal of the American Chemical Society 102, 7816-7817 (1980).

${ }^{2}$ S. Narayan, J. Muldoon, M. Finn, V. V. Fokin, H. C. Kolb, and K. B. Sharpless, "“'on water": unique reactivity of organic compounds in aqueous suspension," Angewandte Chemie International Edition 44, 3275-3279 (2005).

${ }^{3}$ K. Liu, H. Qi, R. Dong, R. Shivhare, M. Addicoat, T. Zhang, H. Sahabudeen, T. Heine, S. Mannsfeld, U. Kaiser, et al., "On-water surface synthesis of crystalline, few-layer two-dimensional polymers assisted by surfactant monolayers," Nat. Chem. 11, 994-1000 (2019).

${ }^{4}$ M. De Rosa, P. La Manna, A. Soriente, C. Gaeta, C. Talotta, and P. Neri, "Exploiting the hydrophobicity of calixarene macrocycles for catalysis under "on-water" conditions," RSC Adv. 6, 91846-91851 (2016).

${ }^{5}$ P. Norcott, C. Spielman, and C. S. McErlean, "An in-water, on-water domino process for synthesis," Green Chem. 14, 605-609 (2012).

${ }^{6}$ K. Piradashvili, E. M. Alexandrino, F. R. Wurm, and K. Landfester, "Reactions and polymerizations at the liquid-liquid interface," Chem. Rev. 116, 2141-2169 (2016).

${ }^{7}$ M. De Rosa, P. La Manna, A. Soriente, C. Gaeta, C. Talotta, N. Hickey, S. Geremia, and P. Neri, "A simple tetraminocalix [4] arene as a highly efficient catalyst under "on-water" conditions through hydrophobic amplification of weak hydrogen bonds," Chem. Eur. J. 23, 7142-7151 (2017).

${ }^{8}$ M. F. Ruiz-Lopez, J. S. Francisco, M. T. Martins-Costa, and J. M. Anglada, "Molecular reactions at aqueous interfaces," Nat. Rev. Chem. 4, 459-475 (2020).

${ }^{9}$ Y. Jung and R. Marcus, "On the theory of organic catalysis "on water", J. Am. Chem. Soc. 129, 5492-5502 (2007).

${ }^{10}$ R. N. Butler and A. G. Coyne, "Water: Nature's reaction enforcercomparative effects for organic synthesis "in-water" and "on-water", Chem. Rev. 110, 6302-6337 (2010).

${ }^{11}$ L. R. Pestana, H. Hao, and T. Head-Gordon, "Diels-alder reactions in water are determined by microsolvation," Nano lett. 20, 606-611 (2019).

${ }^{12}$ R. M. Bain, S. Sathyamoorthi, and R. N. Zare, “"'on-droplet” chemistry: the cycloaddition of diethyl azodicarboxylate and quadricyclane," Angew. Chem. 129, 15279-15283 (2017).
${ }^{13}$ J. K. Beattie, C. S. McErlean, and C. B. Phippen, "The mechanism of onwater catalysis," Chem. Eur. J. 16, 8972-8974 (2010).

${ }^{14}$ T. Kitanosono and S. Kobayashi, "Reactions in water involving the "onwater” mechanism,” Chem. Eur. J. 26, 9408-9429 (2020).

${ }^{15}$ G. Shrivastav, T. S. Khan, M. Agarwal, and M. A. Haider, "A car-parrinello molecular dynamics simulation study of the retro diels-alder reaction for partially saturated 2-pyrones in water," J Phys. Chem. C 122, 11599-11607 (2018).

${ }^{16}$ K. Karhan, R. Z. Khaliullin, and T. D. Kühne, "On the role of interfacial hydrogen bonds in "on-water" catalysis," J. Chem. Phys. 141, 12B632_1 (2014).

${ }^{17}$ M. A. Salem and T. D. Kühne, "Insight from energy decomposition analysis on a hydrogen-bond-mediated mechanism for on-water catalysis," Mol. Phys. 118, e1797920 (2020).

${ }^{18}$ X. Yang, J. Zou, Y. Wang, Y. Xue, and S. Yang, "Role of water in the reaction mechanism and endo/exo selectivity of 1,3-dipolar cycloadditions elucidated by quantum chemistry and machine learning," Chem. Eur. J. 25, 8289-8303 (2019).

${ }^{19}$ O. Sereda, N. Clemens, T. Heckel, and R. Wilhelm, "Imidazolinium and amidinium salts as lewis acid organocatalysts," Beilstein J. Org. Chem. 8, 1798-1803 (2012).

${ }^{20}$ M. Elstner, D. Porezag, G. Jungnickel, J. Elsner, M. Haugk, T. Frauenheim, S. Suhai, and G. Seifert, "Self-consistent-charge density-functional tightbinding method for simulations of complex materials properties," Phys. Rev. B 58, 7260-7268 (1998).

${ }^{21}$ T. D. Kühne, M. Iannuzzi, M. Del Ben, V. V. Rybkin, P. Seewald, F. Stein, T. Laino, R. Z. Khaliullin, O. Schütt, F. Schiffmann, et al., "Cp2k: An electronic structure and molecular dynamics software package-quickstep: Efficient and accurate electronic structure calculations," J. Chem. Phys. 152, 194103 (2020).

${ }^{22}$ T. D. Kühne, M. Krack, F. R. Mohamed, and M. Parrinello, "Efficient and accurate car-parrinello-like approach to born-oppenheimer molecular dynamics," Phys. Rev. Lett. 98, 066401 (2007).

${ }^{23}$ D. Richters and T. D. Kühne, "Self-consistent field theory based molecular dynamics with linear system-size scaling," J. Chem. Phys. 140, 134109 (2014).

${ }^{24}$ M. Doemer, E. Liberatore, J. M. Knaup, I. Tavernelli, and U. Rothlisberger, "In situ parameterisation of scc-dftb repulsive potentials by iterative boltzmann inversion," Molecular Physics 111, 3595-3607 (2013).

${ }^{25}$ S. Kumar, J. M. Rosenberg, D. Bouzida, R. H. Swendsen, and P. A. Kollman, "The weighted histogram analysis method for free-energy calculations on biomolecules. i. the method," Journal of computational chemistry 13, 1011-1021 (1992).

${ }^{26}$ A. Grossfield, "Wham: the weighted histogram analysis method," version 2.0.10.2, http://membrane.urmc.rochester.edu/wordpress/ ?page_id=126. (2020).

${ }^{27} \mathrm{~J}$. VandeVondele and J. Hutter, "An efficient orbital transformation method for electronic structure calculations," The Journal of chemical physics 118, 4365-4369 (2003).

${ }^{28}$ J. E. Argüello, R. Pérez-Ruiz, and M. A. Miranda, "Novel [4+ 2] cycloaddition between thiobenzophenone and aryl-substituted alkenes via photoinduced electron transfer," Org. Lett. 9, 3587-3590 (2007).

${ }^{29}$ R. Bastin, H. Albadri, A.-C. Gaumont, and M. Gulea, "Pyridinedithioesters as heterodienophiles: application to the synthesis of aprikalim," Organic letters 8, 1033-1036 (2006).

${ }^{30}$ R. S. Mulliken, "Electronic population analysis on lcao-mo molecular wave functions. i," J. Chem. Phys. 23, 1833-1840 (1955).

${ }^{31}$ T. A. Manz and D. S. Sholl, "Chemically meaningful atomic charges that reproduce the electrostatic potential in periodic and nonperiodic materials," J. Chem. Theo. Comp. 6, 2455-2468 (2010).

${ }^{32}$ S. K. Sahoo, J. Heske, S. Azadi, Z. Zhang, N. V. Tarakina, M. Oschatz, R. Z. Khaliullin, M. Antonietti, and T. D. Kühne, "on the possibility of helium adsorption in nitrogen doped graphitic materials," Sci. Rep. 10, 1-9 (2020).

${ }^{33} \mathrm{P}$. Li, F. Liu, Y. Shao, and Y. Mei, "Computational insights into endo/exo selectivity of the diels-alder reaction in explicit solvent at ab initio quantum mechanical/molecular mechanical level," J Phys. Chem. B 123, 5131-5138 (2019).

${ }^{34}$ Z. Yang, C. Doubleday, and K. Houk, "QM/QM protocol for direct molecular dynamics of chemical reactions in solution: The water-accelerated 
diels-alder reaction," J. Chem. Theory Comput. 11, 5606-5612 (2015).

${ }^{35}$ F. Liu, Z. Yang, Y. Mei, and K. Houk, "QM/QM' direct molecular dynamics of water-accelerated diels-alder reaction," J. Phys. Chem. B 120, 6250-6254 (2016).

${ }^{36}$ J. Soto-Delgado, R. A. Tapia, and J. Torras, "Multiscale treatment for the molecular mechanism of a diels-alder reaction in solution: A qm/mm-md study," J. Chem. Theory Comput. 12, 4735-4742 (2016).

${ }^{37}$ E. Poli, K. H. Jong, and A. Hassanali, "Charge transfer as a ubiquitous mechanism in determining the negative charge at hydrophobic interfaces," Nature communications 11, 1-13 (2020). 